\title{
Modeling the Competition Between Viruses in a Complex Plant-Pathogen System
}

\author{
Marcos Amaku, Marcelo Nascimento Burattini, Francisco Antonio Bezerra Coutinho, and Eduardo Massad
}

First author: School of Veterinary Medicine, University of São Paulo, São Paulo, SP, Brazil; second, third, and fourth authors: School of Medicine, University of São Paulo and LIM 01-HCFMUSP, São Paulo, SP, Brazil; and fourth author: London School of Hygiene and Tropical Medicine, UK.

Accepted for publication 4 June 2010.

\begin{abstract}
Amaku, M., Burattini, M. N., Coutinho, F. A. B., and Massad, E. 2010. Modeling the competition between viruses in a complex plant-pathogen system. Phytopathology 100:1042-1047.

In this article, we propose a mathematical model that describes the competition between two plant virus strains (MAV and PAV) for both the host plant (oat) and their aphid vectors. We found that although PAV is transmitted by two aphids and MAV by only one, this fact, by itself, does

during the period from 1961 through 1976; an interpretation that is in agreement with the theories of A. G. Power. Also, although MAV wins the competition within aphids, we assumed that, in 1961, PAV mutated into a new variant such that this new variant was able to overcome MAV within the plants during a latent period. As shown below, this is sufficient to explain the swap of strains; that is, the dominant MAV was replaced by $\mathrm{PAV}$, also in agreement with Power's expectations.
\end{abstract} not explain the complete replacement of MAV by PAV in New York State
Additional keywords: virus competition.
Previously, Rochow (14) reported the substitution of the Barley yellow dwarf virus (BYDV) strain MAV by the virus strain PAV in spring oat during the period from 1960 until 1975. This was interpreted by Power (12) as a competition between two virus strains, with the strain PAV winning the competition.

This article aims to formalize Power's arguments by providing a mathematical model for such competition that explains how the virus strain MAV could be replaced by the PAV strain.

The article by Power distinguishes between two types of competition: competition within the host (for the cells of the host) is called internal competition, and competition for infection of the hosts among the population is called external competition.

An attempt to model this phenomenon was made by Zhang and Holt (16). However, their model does not include vectors, rendering it less complete than the present model. The purpose of this article is to model Power's ideas, including two vectors and internal and external competitions, with a mathematical model that describes the exclusion of one virus strain by the other in quantitative terms.

The article is organized as follows. In the next section, we present the equations of the model and review the types of competition that are implied by the equations. Then, we discuss and justify the parameters based on data from the literature. Finally, we present the results of the model and, in the final section, we draw conclusions from our study, including some implied practical consequences.

\section{THEORY AND APPROACHES}

Competition between plant viruses according to Power. The prevalence of the BYDV strains PAV and MAV recovered from oat plants collected in New York is shown in Figure 1. It can be

Corresponding author: M. Amaku; E-mail address: amaku@usp.br

doi:10.1094/PHYTO-10-09-0289

(C) 2010 The American Phytopathological Society seen that PAV replaced MAV in the 18-year period from 1957 to 1975 and that MAV infection was effectively eliminated as its prevalence declined from 90 to $0 \%$ while the PAV infection rose from 3 to $98 \%$. The remaining $2 \%$ are plants infected by other strains (14).

The virus strains PAV and MAV are transmitted to oat plants by aphid vectors. The PAV virus is transmitted by two species of aphid vectors, Sitobion avenae and Rhopalosiphum padi, but the MAV strain is only transmitted by $S$. avenae. As noted by Power (12), "on the surface PAV has more opportunities for transmission and could be expected to increase." However, as we will demonstrate, only competition can explain why MAV almost disappeared, in spite of the fact that the abundance of both vectors remained constant over the period (14).

Competition among virus strains within the host plant or vector may affect the success of a virus strain (16). Competition may occur within the plant for replication sites and within the vector for transmission sites. We have no evidence of competition within the vector for propagation sites. Note that, because the competition mechanism is not the same in the host and vector, the stronger competitor within the host plant will not necessarily be the stronger competitor for the vectors.

Competition between the two BYDV strains within plants is described by Power (12) as follows. If a host plant acquires PAV and MAV at the same time, PAV interferes with the replication of MAV, leading to a lower MAV concentration within the phloem of the host plant (15). As a result, there is a lower probability of MAV being acquired and transmitted to the aphid (5). This suggests that, in direct competition within the host plant, PAV is the stronger competitor. However, if a plant acquires either strain at least 15 days before the other, then the second strain undergoes a comparatively small amount of replication. Thus, PAV dominates MAV within the plant in simultaneous infections only if both infection events occur within the latent period of an existing MAV infection; which we assume to be 15 days, based on the experimental data given by Gray et al. (5). Once one strain is established within the plant, the plant is resistant to invasion by the other. 
On the other hand, within the vector ( $S$. avenae), MAV inhibits the transmission of PAV, apparently via saturation of the sites in the salivary glands that allow transmission to occur (4). Therefore, within the vector, MAV outcompetes PAV.

Another facet of the competition dynamics is the efficiency of transmission. A number of experiments have been reported that examined the feeding times required for an aphid to acquire a virus from an infected plant $(5,13)$. The conclusions of these reports are that $S$. avenae transmits MAV much more efficiently $(82.0 \%)$ than it transmits PAV (53.3\%), and $R$. padi transmits PAV $(86.7 \%)$ as efficiently as $S$. avenae transmits MAV. Thus, a decline in MAV is unexpected solely because PAV is transmitted by two aphids ( $S$. avenae and $R$. padi) and MAV is transmitted by only $S$. avenae.

The mathematical model given below quantitatively predicts the disappearance of MAV due to competition within the host plant.

Because MAV is more efficient within the aphid $S$. avenae, it is not clear that PAV can displace MAV, even though it is transmitted by two vectors. Therefore, a more realistic quantitative approach, in which we gradually introduce biological realities, is required.

The model. The model considers plants (hosts), insects (vectors), and parasites (virus strains). Host plants are divided into susceptible plants $S_{h}(t)$, latent plants $L_{h 1}(t)$ (infected by PAV strain) and $L_{h 2}(t)$ (infected by MAV strain), and infected plants $I_{h 1}(t)$ (infected by PAV strain) and $I_{h 2}(t)$ (infected by MAV strain). The latent period is denoted by $1 / \gamma$, where $\gamma$ is the transmission rate from the latent to the infectious state. Susceptible plants are bitten by the vectors $S$. avenae and $R$. padi with the rates $a_{v}$ and $a_{w}$, respectively. The susceptible plants are infected with probabilities $b_{v 1}, b_{v 2}$, and $b_{w 1}$, where $b_{v 1}$ is the probability of transmission of PAV by $S$. avenae, $b_{v 2}$ is the probability of transmission of MAV by $S$. avenae, and $b_{w 1}$ is the probability of transmission of PAV by $R$. padi. In addition, plants have the mortality rates $\mu_{h}$ (natural mortality) and $\alpha_{h}$ (disease-induced mortality). For simplicity, $\alpha_{h}$ is considered as equal for both virus strains. This induced mortality is not very important (Parras, personal communication). The plants change their aspect when infected but do not appear to die in a much faster rate.

The $S$. avenae vectors are classified as susceptible vectors, $S_{v}(t)$; vectors infected by PAV, $I_{h 1}(t)$; and vectors infected by MAV, $I_{h 2}(t)$. The vectors are subject to a natural mortality rate, which is denoted by $\mu_{v}$. The $R$. padi vectors are divided into susceptible vectors, $S_{w}(t)$ and vectors infected by PAV, $I_{w 1}(t)$. These vectors are also subject to a natural mortality rate, which is denoted by $\mu_{w}$. It is important to note again that $S$. avenae transmits both strains of virus (MAV and PAV) but $R$. padi transmits only PAV.

The susceptible host plants acquire infection from infected aphids through the terms

$$
a_{v} b_{v 1} I_{v 1}(t) \frac{S_{h}(t)}{N_{h}(t)}, a_{v} b_{v 2} I_{v 2}(t) \frac{S_{h}(t)}{N_{h}(t)}, \text { and } a_{w} b_{w 1} I_{w 1}(t) \frac{S_{h}(t)}{N_{h}(t)}
$$

Let us consider the first term. The number of bites inflicted per unit time by the first aphid (S. avenae) is $a_{v} I_{v 1}(t)$. The fraction of those bites on susceptible plants is

$$
a_{v} I_{v 1}(t) \frac{S_{h}(t)}{N_{h}(t)}
$$

and a proportion, $b_{\mathrm{v} 1}$, of those bites results in infection. Therefore, the first term

$$
a_{v} b_{v 1} I_{v 1}(t) \frac{S_{h}(t)}{N_{h}(t)}
$$

is the number of susceptible plants infected by the PAV strain through $S$. avenae per unit time. Similarly, the term

$$
a_{v} b_{v 2} I_{v 2}(t) \frac{S_{h}(t)}{N_{h}(t)}
$$

is the number of susceptible plants that get infected by the MAV strain by $S$. avenae per unit time, and the term

$$
a_{w} b_{w 1} I_{w 1}(t) \frac{S_{h}(t)}{N_{h}(t)}
$$

is the number of susceptible plants that get infected by the PAV strain by $R$. padi per unit time.

Drawing these points together, we can specify the equations for the plant hosts as shown below (equation system 1). In the equations for the vectors, the terms

$$
a_{v} c_{v} S_{v}(t) \frac{I_{h 1}(t)}{N_{h}(t)}, a_{v} c_{v} S_{v}(t) \frac{I_{h 2}(t)}{N_{h}(t)} \text { and } a_{w} c_{w} S_{w}(t) \frac{I_{h 1}(t)}{N_{h}(t)}
$$

represent the number of vectors that get infected by biting infected host plants. In the first term, $a_{v} S_{v}(t)$ is the number of bites that the susceptible vector $S$. avenae $\left(S_{v}\right)$ gives per unit time. Of those, a fraction of bites

$$
a_{v} S_{v}(t) \frac{I_{h 1}(t)}{N_{h}(t)}
$$

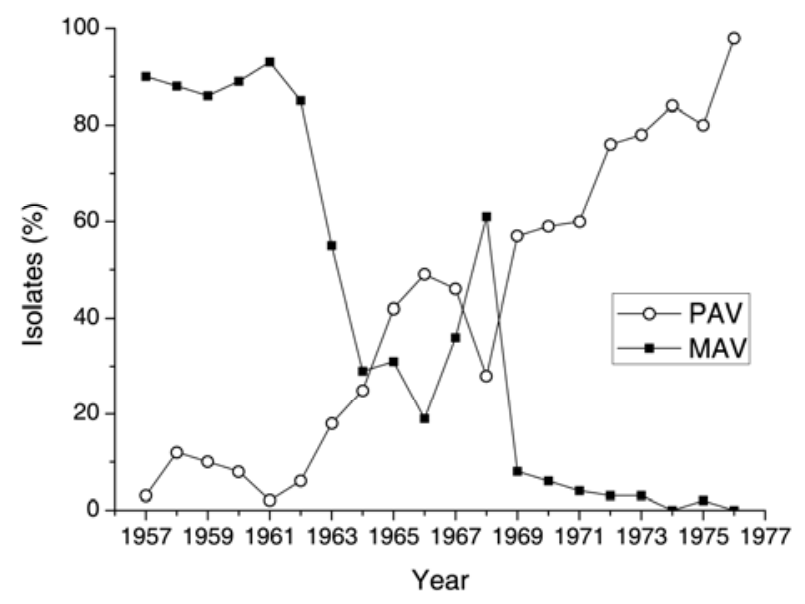

Fig. 1. Percentage of virus isolates (PAV and MAV strains) recovered from oat plants collected in New York State over a period of 20 years (14).

$$
\begin{aligned}
& \frac{d S_{h}(t)}{d t}=-a_{v} b_{v 1} I_{v 1}(t) \frac{S_{h}(t)}{N_{h}(t)}-a_{v} b_{v 2} I_{v 2}(t) \frac{S_{h}(t)}{N_{h}(t)}-a_{w} b_{w 1} I_{w 1}(t) \frac{S_{h}(t)}{N_{h}(t)}-\left(\mu_{h}+h(t)\right) S_{h}(t)+\Lambda_{h}(t) \\
& \frac{d L_{h 1}(t)}{d t}=a_{v} b_{v 1} I_{v 1}(t) \frac{S_{h}(t)}{N_{h}(t)}+a_{w} b_{w 1} I_{w 1}(t) \frac{S_{h}(t)}{N_{h}(t)}+z_{h} a_{v} b_{v 1} I_{v 1}(t) \frac{L_{h 2}(t)}{N_{h}(t)}+z_{h} a_{w} b_{w 1} I_{w 1}(t) \frac{L_{h 2}(t)}{N_{h}(t)}-\gamma L_{h 1}(t)-\left(\alpha_{h}+h(t)\right) L_{h 1}(t) \\
& \frac{d L_{h 2}(t)}{d t}=a_{v} b_{v 2} I_{v 2}(t) \frac{S_{h}(t)}{N_{h}(t)}-z_{h} a_{v} b_{v 1} I_{v 1}(t) \frac{L_{h 2}(t)}{N_{h}(t)}-z_{h} a_{w} b_{w 1} I_{w 1}(t) \frac{L_{h 2}(t)}{N_{h}(t)}-\gamma L_{h 2}(t)-\left(\alpha_{h}+h(t)\right) L_{h 2}(t) \\
& \frac{d I_{h 1}(t)}{d t}=\gamma L_{h 1}(t)-\left(\alpha_{h}+h(t)\right) I_{h 1}(t) \\
& \frac{d I_{h 2}(t)}{d t}=\gamma L_{h 2}(t)-\left(\alpha_{h}+h(t)\right) I_{h 2}(t)
\end{aligned}
$$




$$
\begin{aligned}
& \frac{d S_{v}(t)}{d t}=-a_{v} c_{v} S_{v}(t) \frac{I_{h 1}(t)}{N_{h}(t)}-a_{v} c_{v} S_{v}(t) \frac{I_{h 2}(t)}{N_{h}(t)}-\left(\mu_{v}+h(t)\right) S_{v}(t)+\eta\left[I_{v 1}(t)+I_{v 2}(t)\right]+\Lambda_{v}(t) \\
& \frac{d I_{v 1}(t)}{d t}=a_{v} c_{v} S_{v}(t) \frac{I_{h 1}(t)}{N_{h}(t)}-z_{v} a_{v} c_{v} I_{v 1}(t) \frac{I_{h 2}(t)}{N_{h}(t)}-\left(\eta+\mu_{v}+h(t)\right) I_{v 1}(t) \\
& \frac{d I_{v 2}(t)}{d t}=a_{v} c_{v} S_{v}(t) \frac{I_{h 2}(t)}{N_{h}(t)}+z_{v} a_{v} c_{v} I_{v 1}(t) \frac{I_{h 2}(t)}{N_{h}(t)}-\left(\eta+\mu_{v}+h(t)\right) I_{v 2}(t) \\
& \frac{d S_{w}(t)}{d t}=-a_{w} c_{w} S_{w}(t) \frac{I_{h 1}(t)}{N_{h}(t)}-\left(\mu_{w}+h(t)\right) S_{w}(t)+\eta I_{w 1}(t)+\Lambda_{w}(t) \\
& \frac{d I_{w 1}(t)}{d t}=a_{w} c_{w} S_{w}(t) \frac{I_{h 1}(t)}{N_{h}(t)}-\left(\eta+\mu_{w}+h(t)\right) I_{w 1}(t)
\end{aligned}
$$

are on hosts infected with PAV strain

$$
\left(\frac{I_{h 1}}{N_{h}}\right)
$$

and a proportion, $c_{v}$, gets infected. Therefore, the term

$$
a_{v} c_{v} S_{v}(t) \frac{I_{h 1}(t)}{N_{h}(t)}
$$

is the number of susceptible $S$. avenae vectors that get infected with the PAV strain per unit time. The other two terms can be interpreted in the same manner.

When the recovery rate to the susceptible state, $\eta$, is equal for both vectors, the system of equations for the vectors is given by equation system 2 . In addition to the mortality rates, the populations suffer harvesting (a human-induced mortality) given by:

$$
h(t)=\sum_{n=0}^{\infty} H \theta\left(t-\left(t_{i}+n\right)\right) \theta\left(\left(t_{i}+n\right)+\Delta t_{i}-t\right)
$$

where $H$ is the rate of harvesting (a constant), and $\theta\left[t-\left(t_{i}+n\right)\right]$ and $\theta\left[\left(t_{i}+n\right)+\Delta t_{i}-t\right]$ are Heaviside functions. In the simulations described below, we take $t_{\mathrm{i}}$ as the first harvesting time and $\Delta t_{i}$ as the duration of harvesting, such that $\Delta t_{i}<t_{i}$, and $n$ is the number of years.

The term $\Lambda_{\mathrm{h}}$ is a reposition term for the plants that die or are harvested and, thus, includes sowing, $h\left(t-\Delta t_{i}\right)$, to compensate for harvesting. This term was included to keep the population of plants constant. This is, of course, an oversimplification of the regulation mechanism of the size of the plant system, assumed to be done by the farmers, and is the simplest way of modeling it. We are going to assume that the sowing time begins immediately after the harvesting time and that the two periods have the same duration. Moreover, we assume that the reposition occurs immediately into the susceptible state, so that the maturation of the plant from seed to adults is not considered. Thus, we have:

$$
\begin{aligned}
& \Lambda_{h}(t)=\left(\mu_{h}+h\left(t-\Delta t_{i}\right)\right) S_{h}(t)+\left(\alpha_{h}+h\left(t-\Delta t_{i}\right)\right) \times \\
& {\left[L_{h 1}(t)+L_{h 2}(t)+I_{h 1}(t)+I_{h 2}(t)\right]}
\end{aligned}
$$

The assumed reposition terms $\Lambda_{v}$ and $\Lambda_{w}$ keep the vector population constant, which is a convenient simplification. Note that we are considering the effect of harvest on the vector populations through the term $h(t)$ in the system of equation 2. By considering that harvest affects the vector population in the same way as it affects the plant population, we are neglecting the socalled diapause effect. Therefore, we may be overestimating this effect. However, this is admissible because numerical simulations show that this harvest has a negligible effect (because the vector population recovers very fast) on the outcome of the model.

$$
\begin{gathered}
\Lambda_{v}(t)=\left[\mu_{v}+h\left(t-\Delta t_{i}\right)\right]\left[S_{v}(t)+I_{v 1}(t)+I_{v 2}(t)\right] \\
\left.\Lambda_{w}(t)=\left[\mu_{w}+h\left(t-\Delta t_{i}\right)\right)\right]\left[S_{w}(t)+I_{w 1}(t)\right]
\end{gathered}
$$

The competition between viruses manifests itself in several ways. Let us first consider the host plants. We assume that, if a plant is infected by a virus strain, it passes a latent period and then becomes immune to the other strain. On the other hand, when a plant host infected by MAV is bitten by a vector infected with PAV during the MAV latent period, it becomes infected with PAV only. This is assumed for simplicity to occur instantaneously. This simplification could be avoided by introducing a new compartment with plants infected by the two strains simultaneously. However, this compartment may be considered negligible due to the very short interval plants remain in it. In fact, according to Wen et al. (15), low concentrations of one virus strain in the phloem results in low transmission of this strain. These considerations are included in the model by the term

$$
z_{h} a_{v} b_{v 1} I_{v 1}(t) \frac{L_{h 2}(t)}{N_{h}(t)}+z_{h} a_{w} b_{w 1} I_{w 1}(t) \frac{L_{h 2}(t)}{N_{h}(t)}
$$

which has a minus sign in the third equation and a plus sign in the second equation of system 1 . We have included the parameter $z_{h}$ which is normally taken to be 1 but can also equal 0 when we want to exclude this type of competition. When we exclude this type of competition, there can still be competition that arises because each plant can be infected by only one strain: the first strain to infect. We call this basic competition, which does not consider the competition within vectors, as described below.

Now consider the competition within the vectors. When an $S$. avenae vector that is infected with PAV bites a plant infected with MAV, it becomes instantaneously infected by MAV, which wins the internal competition within the vector. This is modeled by the term

$$
z_{v} a c I_{v 1}(t) \frac{I_{h 2}(t)}{N_{h}(t)}
$$

which has a minus sign in the second equation of system 2 and a plus sign in the third equation of system 2 . We have included a parameter $z_{v}$, which is normally taken to be 1 but can be put equal to 0 when we want to exclude this type of competition. Thus, if $z_{v}=1$ in the latent period, MAV loses the internal competition within the host.

A crucial aspect of the model is that MAV wins the competition in the vectors but loses the competition in the plant (12), as explained above.

The model's parameters, defined in Table 1, were obtained from several references. To solve the differential equations numerically, we used the fourth-order Runge-Kutta method with automatic step size control to maintain accuracy.

\section{RESULTS}

Simulating the model. First, we only consider basic competition: each plant can be infected only by the strain that infects it first (16). Therefore, we set $z_{h}=0$ and $z_{v}=0$. The aim of this simulation is to investigate whether PAV can eliminate MAV simply because it is transmitted by two vectors, a possibility that was rejected by Power (12). Using the parameters given in Table 1 , we verify that basic competition by itself does not eliminate MAV, which is in agreement with Power's expectations (12), as shown in the Appendix.

Now, simulating the model with full competition-that is, $z_{h}=$ 1 and $z_{v}=1-$ we see that MAV can be completely eliminated, 
TABLE 1. Notation, biological meaning, value, and source of the parameters used in the model

\begin{tabular}{|c|c|c|c|}
\hline Notation & Biological meaning & Value & Source, comment \\
\hline$a_{v}$ & Bite rate of Sitobion avenae & 2 day $^{-1}=730$ year $^{-1}$ & Power (12) \\
\hline$a_{w}$ & Bite rate of Rhopalosiphum padi & 2 day $^{-1}=730$ year $^{-1}$ & Power (12) \\
\hline$b_{v 1}$ & Probability of transmission of PAV by $S$. avenae & 0.533 & Power (12) \\
\hline$b_{v 2}$ & Probability of transmission of MAV by S. avenae & 0.820 & Power (12) \\
\hline$b_{w 1}$ & Probability of transmission of PAV by $R$. padi & 0.867 & Power (12) \\
\hline$c_{v}$ & Susceptibility of $S$. avenae & 0.09 & Fitted \\
\hline$c_{w}$ & Susceptibility of $R$. padi & 0.09 & Fitted \\
\hline$\mu_{v}$ & S. avenae mortality rate & 24.3 year $^{-1}$ & Lopes $^{\mathrm{a}}$ \\
\hline$\mu_{w}$ & R. padi mortality rate & 24.3 year $^{-1}$ & Lopes $^{\text {a }}$ \\
\hline$\eta$ & Vector recovery rate & 52 year $^{-1}$ & Lopes $^{\mathrm{a}}$ \\
\hline$\mu_{h}$ & Host plant mortality & 2.4 year $^{-1}$ & Lopes $^{\mathrm{a}}$ \\
\hline $1 / \gamma$ & Latent period & 15 days & Power (12) \\
\hline
\end{tabular}

a J. R. S. Lopes, personal communication.

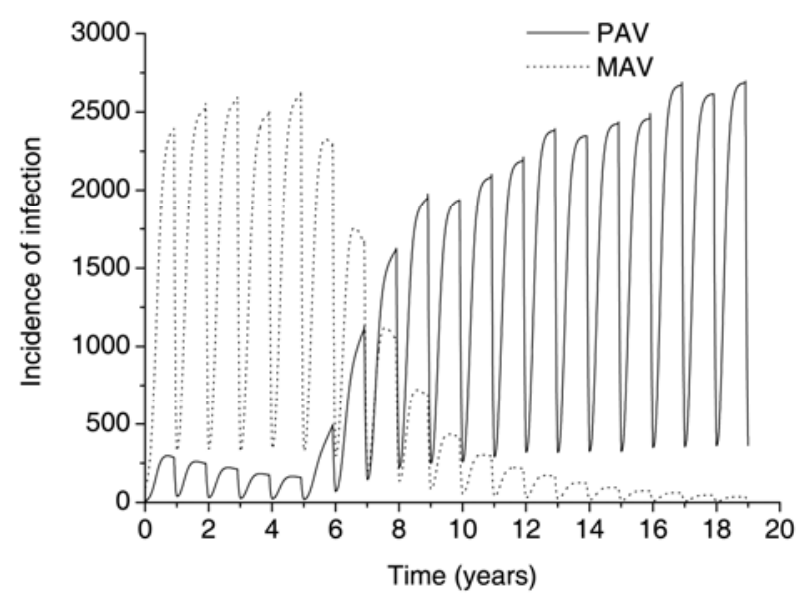

Fig. 2. Number of host plants infected with PAV or MAV strains over time, assuming that: $N_{\mathrm{h}}(0)=500, N_{\mathrm{v}}(0)=20$, and $N_{\mathrm{w}}(0)=10$ for the initial number of host plants, Sitobion avenae vectors, and Rhopalosiphum padi vectors, respectively; an initial percentage of $80 \%$ susceptible and $20 \%$ infected host plants and vectors; $H=24$ year $^{-1} ; t_{\mathrm{i}}=11 / 12=0.92$ year; and $\Delta t_{\mathrm{i}}=1 / 12=0.083$ year.

which is in agreement with the data. The proportions of MAV and PAV were calculated by integrating the number of plants harvested during each harvest period and dividing by the total (see equation 7 below).

In order to simulate the substitution of MAV by PAV, we assumed that MAV was the dominant strain until 1961. Then, we assumed that, in 1961, PAV mutated into a new variant such that this new variant was able to overcome MAV within the plants during the latent period. The simulation included a new term,

$$
\left(1-z_{h}\right) a_{v} b_{v 2} I_{v 2}(t) \frac{L_{h 1}(t)}{N_{h}(t)},
$$

leading to $z_{h}=\theta(t-5)$, (where $\left.t=0=1957\right)$, in the second and third equations of system 1 . This term means that, until $1961(t=$ 5), $z_{h}=0$ and the term

$$
a_{v} b_{v 2} I_{v 2}(t) \frac{L_{h 1}(t)}{N_{h}(t)}
$$

guarantees that MAV dominates by replacing PAV in the latent period. After $1961(t=5), z_{h}=1$ and PAV starts to replace MAV in the plants during the latent period. Note that MAV still wins the competition within the vector and that such a small modification (that is, PAV wins the competition within the plants only during the latent period) changes the dominance of the strains.

The result of the simulation is presented in Figures 2 and 3, which show the oscillatory harvesting pattern (Fig. 2) and PAV replacing MAV (Fig. 3). In Figure 2, we note the effect of harvesting starting at $t_{i} \approx 0.92$ years (last month of the 12-month harvesting period), where there is a trough in both population numbers. These troughs repeat annually $\left(t_{i}+1, t_{i}+2, \ldots\right)$ and we can see PAV increasing and MAV decreasing, with the two

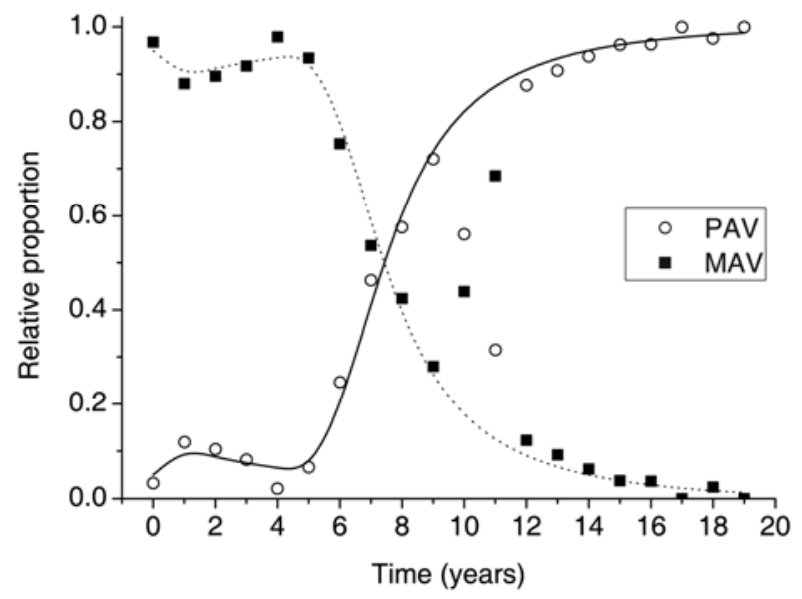

Fig. 3. Relative proportions of PAV and MAV strains over a period of 20 years and the result of the simulations for PAV (solid line) and MAV (dotted line), assuming that: $N_{\mathrm{h}}(0)=500, N_{\mathrm{v}}(0)=20$, and $N_{\mathrm{w}}(0)=10$ for the initial number of host plants, Sitobion avenae vectors and Rhopalosiphum padi vectors, respectively; an initial percentage of $80 \%$ susceptible and $20 \%$ infected host plants and vectors; $H=24$ year $^{-1} ; t_{\mathrm{i}}=11 / 12=0.92$ year; and $\Delta t_{\mathrm{i}}=1 / 12=$ 0.083 year. The result of the simulations are, of course, points. However, in the figure, we show lines connecting them.

populations crossing between the seventh and the eighth years. Note that plants survive between two cropping seasons. This is due to a limitation of the model, which results from the fact that we are assuming a constant harvest effort (a constant rate $H$ ). This results in an exponential decay in the number of plants that tends to zero only asymptotically. Because this model is deterministic, this effect cannot be avoided. However, it is negligible to the outcome of the model. We have assumed a harvest rate $H=24$ year $^{-1}$, which should be enough to wipe out the plant population in 2 weeks; however, as can be observed, there is always a residual fraction that survives.

The replacement of MAV by PAV is also shown in Figure 3, which shows the proportion of harvested plants infected with MAV (squares) and PAV (circles). This calculation was performed by integrating the mortality of the plants during the harvest interval. The proportion of harvested plants infected by PAV is given by:

$$
\frac{\int_{t_{i}+n}^{t_{i}+n+1} H \times\left(L_{h 1}(t)+I_{h 1}(t)\right) d t}{\int_{t_{i}+n}^{t_{i}+n+1} H \times\left(L_{h 1}(t)+I_{h 1}(t)\right) d t+\int_{t_{i}+n}^{t_{i}+n+1} H \times\left(L_{h 2}(t)+I_{h 2}(t)\right) d t}, n=0,1,2 \ldots
$$

A similar relationship gives the proportion of harvested plants infected by MAV. Note that the harvesting rate $(H)$ cancels out. 
Note that, in Figure 2, the initial number of plants and vectors, which were arbitrarily chosen, determine the maximum values attained by each population.

The substitution of PAV by MAV in the aphid and the substitution of MAV by PAV in the latent period in the plant host are experimentally demonstrated facts $(12,14)$. The mutation hypothesis is based on the observation by Rochow (14), who stated that: "an obvious possibility is that the change from MAVlike isolates to PAV-like ones simply reflects a change in predominance of aphid species from $M$. avenae to $R$. padi but our observations of vectors pathogen types and disease incidence in small grains during each of these years do not support this explanation." Because MAV obviously continued to dominate PAV within the vector, a plausible explanation is a mutation in the PAV virus itself. Mutation in the plant cannot be ruled out but it seems very unlikely because PAV causes more damage to the plants (14). In any event, the result would be the same since the model would be unchanged.

\section{DISCUSSION}

Our approach was based on the data analyzed by Power (12) and her description of the competition between two virus strains for one host plant. The articles by Power (12) and Rochow (14) give an unusually thorough study of a competition process between two pathogenic virus strains.

An attempt to model this phenomenon was made by Zhang and Holt (16). However, their model does not include vectors, rendering it less complete than the present model. Our model includes two vectors, one of which transmits only one of the pathogens.
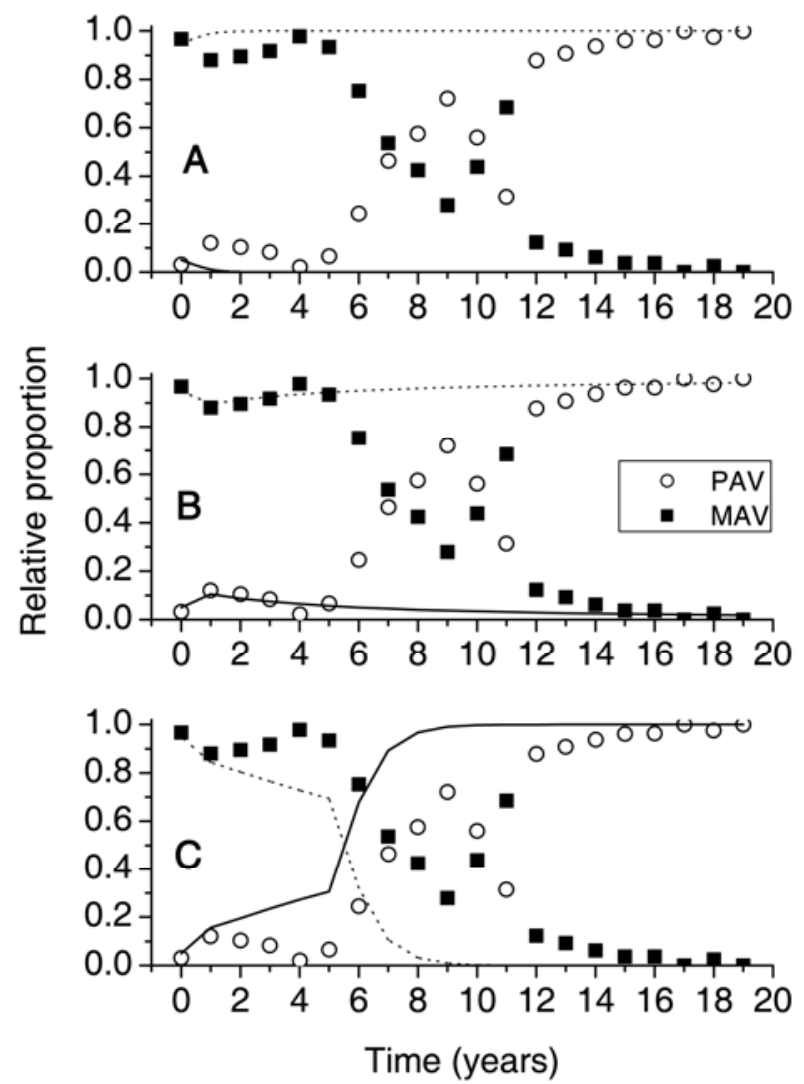

Fig. 4. Relative proportions of PAV and MAV strains over a period of 20 years and the result of the simulations for PAV (solid line) and MAV (dotted line), assuming all the parameters and initial conditions used in Figure 3, for three different variants of the original model (see text for details): $\mathbf{A}$, removing the Rhopalosiphum padi vector population; B, removing the competitive superiority of PAV in the plant; and $\mathbf{C}$, removing the competitive superiority of MAV in the vector.
Also, in this work, a more detailed discussion of virus competition, following closely the findings of Rochow (14) and Power (12), is included. Thus, this model allows for a close examination of the whole system according to the ideas of Power (12). Of course, Zhang and Holt's model (16) and other articles cited there may apply to a larger class of viral infection of plants.

The competition between the two virus strains for a single host has important consequences for host-parasite dynamics in nature (10); in particular, this information may be relevant to certain aspects of medical sciences, such as the dynamic antibiotic resistance $(1,9,10)$.

It is worthwhile to describe a few diseases for which virus competition can be important. This description can be found in the article by Zhang and Holt (16). They describe experimental observations that some viruses offer protection against the invasion of other viruses. In addition, in the article by Dib et al. (3), there are examples of intracellular pathogens that prevent coinfections, such as Babesia bovis, Anaplasma marginale, and Theileria orientalis.

From a theoretical point of view, viral evolution and competition have been addressed by several authors $(1,2,6-9,11)$.

In this article, as mentioned before, we proposed a mathematical model to formalize Power's analysis of the competition between the virus strains PAV and MAV for both the host plant (oat) and the aphid vectors (12). We found that, although PAV is transmitted by two aphids and MAV by just one, this fact, by itself, does not explain the observed replacement of MAV by PAV in New York State between 1961 and 1976. Our model formalizes Power's inferences about virus competition, based on biological mechanism, and shows that the observed replacement event is consistent with the proposed mechanism. Also, although MAV wins the competition within the aphids, we found that a change in the biology, such that PAV becomes the winning strain within the plants (in the latent period), was sufficient to explain the replacement phenomenon; that is, the dominant strain MAV was replaced by PAV, also in agreement with Power's expectations. One plausible mechanism to induce such a change would be a mutation in the PAV strain. Any model describing two virus strains, two aphid vectors, and one plant host is unlikely to be very simple but the one presented here, we suggest, achieves a useful balance between simplicity, biological insight, and predictive accuracy. The model not only describes the observed data but also quantifies the possible underlying biological mechanism proposed by Power (12) to explain the substitution of one pathogen strain by another.

Finally, we should note that it appears (Fig. 3) that the modeled dynamics are much smoother than the observed dynamics during the critical period (year 5 until year 12) while the "new" competition process takes effect. Thus, although the model does a good job of describing the qualitative patterns in the whole data set, there is some evidence that the actual competition process is sensitive to some important source of noise or, indeed, a further mechanism, that have not been included.

\section{APPENDIX}

Additional model analysis. The model analysis presented in the main text shows that the model produces the correct dynamic trajectories. However, it is interesting to investigate with some more details the relative importance of the mechanisms (suggested by Power [12] and described in the main text) in explaining the replacement of MAV by PAV.

Removing the $\boldsymbol{R}$. padi vector population. For this, we removed the two last equations of system 2 and the terms indexed $w$ in system 1. By keeping all the parameters and initial conditions as in the original simulations, it is observed that MAV immediately reaches $100 \%$ prevalence and PAV disappears at the same time (Fig. 4A). Therefore, we may conclude that the existence of the $R$. padi, which transmits only PAV, is essential to the existence 
of PAV in the system and, thus, is an essential component to explain the coexistence up to year 5 .

Removing the competitive superiority of PAV in the plant. This can be done by setting $z_{h}=0$ in system 1 . By keeping all the parameters and initial conditions as in the original simulations, it is observed that MAV tends to $100 \%$ after some years and PAV tends to zero at the same time (Fig. 4B). Therefore, competitive superiority of PAV in the plant seems to be essential to explain the replacement of MAV by PAV. This is a surprising result because the competitive superiority of PAV in the plant takes place only during a small latent period.

Removing the competitive superiority of MAV in the vector. This can be done by setting $z_{v}=0$ in system 2 . By keeping all the parameters and initial conditions as in the original simulations, it is observed that the replacement of MAV by PAV occurs earlier than that observed in the original simulations (Fig. 4C). This can be easily understood because MAV no longer wins the competition within the vector.

The above analyses strongly suggest that competition within the plant is more important to explain the replacement than the competition within the vector. However, the presence of a second vector ( $R$. padi) is essential to explain the existence of PAV in the first 5 years of the examined period. The above results strongly support Power's qualitative insights that although "PAV can be transmitted by $S$. avenae and $R$. padi...this does not, however, explain the decline of MAV unless direct competition also occurs." Our results strongly suggest that competition within the plant is a more important mechanism than the competition within the vectors.

\section{ACKNOWLEDGMENTS}

We thank J. R. Parra and J. R. S. Lopes of ESALQ-USP for their illuminating advice concerning the biology of the complex aphidsviruses-plants, and the editor and one anonymous reviewer for their very useful suggestion to improve this manuscript. This work was partially supported by LIM 01 HCFMUSP, FAPESP, and CNPq.

\section{LITERATURE CITED}

1. Amaku, M., Burattini, M. N., Coutinho, F. A. B., and Massad, E. 2010. Modeling the dynamics of viral evolution considering competition within individual hosts and at population level: the effects of treatment, Bull. Math. Biol. 72:1294-1314. doi: 10.1007/s11538-009-9495-6.

2. Day, T. 2001. Parasite transmission modes and the evolution of virulence. Evolution 55:2389-2400.

3. Dib, L., Bitam, I., Tahri, M., Bensouilah, M., and De Meeus, T. 2008. Competitive exclusion between piroplasmosis and anaplasmosis agents within cattle. Plos Pathog. 4:7.

4. Gildow, F. E., and Rochow, W. F. 1980. Transmission interference between two isolates of barley yellow dwarf virus in Macrosiphum avenae. Phytopathology 70:122-126.

5. Gray, S. M., Power, A. G., Smith, D. M., Seaman, A. J., and Altman, N. S. 1991. Aphid transmission of barley yellow dwarf virus: acquisition access periods and virus concentration requirements. Phytopathology 81:539545.

6. Lipsitch, M., and Nowak, M. A. 1995. The evolution of virulence in sexually transmitted HIV/AIDS. J. Theor. Biol. 174:427-440.

7. Lipsitch, M., Siller, S., and Nowak, M. A. 1996. The evolution of virulence in pathogens with vertical and horizontal transmission. Evolution 50:1729-1741.

8. Manrubia, S. C., and Lázaro, E. 2006.Viral evolution. Physiol. Life Rev. 3:65-92.

9. Massad, E. 1997. Transmission rates and the evolution of HIV virulence. Evolution 50:916-918.

10. Massad, E., Burattini, M. N., and Coutinho, F. A. B. 2008. An optimization model for antibiotic use. Appl. Math. Comput. 201:161-167.

11. Perelson, A. S., and Nelson, P. W. 1999. Mathematical analysis of HIV-1 dynamics in vivo. SIAM Rev. 41:3-44.

12. Power, A. G. 1996. Competition between viruses in a complex plantpathogen system. Ecology 77:1004-1010.

13. Power, A. G., Seaman, A. J., and Gray, S. M. 1991. Aphid transmission of barley yellow dwarf virus: inoculation access periods and epidemiological implications. Phytopathology 81:545-548.

14. Rochow, W. F. 1979. Field variants of barley yellow dwarf virus: detection and fluctuation during twenty years. Phytopathology 69:655660.

15. Wen, F., Lister, R. M., and Fattouh, F. A. 1991. Cross-protection among strains of barley dwarf virus. J. Gen. Virol. 72:729-799.

16. Zhang, X. S., and Holt, J. 2001. Mathematical models of cross protection in the epidemiology of plant-virus diseases. Phytopathology 91:924-934. 